\title{
Patient, caregiver, and provider perceptions of pain and pain management in adolescents and young adults with bleeding disorders
}

\author{
Angela Lambing $^{1}$ | Cynthia D. Nichols ${ }^{2}$ | James E. Munn ${ }^{3}$ | Terry L. Anderson ${ }^{4}$ | \\ Bartholomew J. Tortella $^{4}$ | Michelle L. Witkop ${ }^{2}$ (D)
}

${ }^{1}$ Henry Ford Hemophilia and Thrombosis Treatment Center, Detroit, MI, USA

${ }^{2}$ Munson Medical Center, Traverse City, MI, USA

${ }^{3}$ University of Michigan Hemophilia Treatment Center, Ann Arbor, MI, USA

${ }^{4}$ Pfizer Inc, Collegeville, PA, USA

Correspondence

Michelle L. Witkop, Northern Regional

Bleeding Disorder Center, Munson Medical

Center, Traverse, MI, USA.

Email: mwitkop@mhc.net

Funding information

Pfizer
Introduction: Recurrent bleeding and associated pain are critical components in the management of bleeding disorders, yet scant data describe perceptions of pain in this patient population.

Objective: This study assessed perceptions of pain and pain management in adolescents and young adults (AYAs) with haemophilia or von Willebrand disease (VWD) to determine agreement/disagreement between patients, caregivers and health care providers.

Methods: Using an online questionnaire, AYA patients $(N=89)$, their caregivers $(N=77)$, and providers $(\mathrm{N}=54)$ reported on pain perception, pain treatment and pain control. Acute and chronic pain was measured in patients via the Faces Pain Scale-Revised (FPS-R). Questionnaires queried about pharmacologic and non-pharmacologic pain management methods and how well providers and caregivers helped to manage pain. Results: Poor agreement existed between patients and caregivers across all pain levels, perception of pain control and effectiveness of pain management. Specifically for chronic pain, poor agreement was noted between patients and caregivers (kappa $=0.04$; $29 \%$ agreement) and patients and providers (kappa $=-0.07 ; 21.4 \%$ agreement). Among patients reporting acute or chronic pain, only $67 \%$ and $43 \%$, respectively, utilized medication for their specific pain. Patients used more opioid medications than expected by their providers. On average, AYAs reported initial use of pain medications for chronic pain at 11.5 years.

Conclusions: Ongoing research is needed in haemophilia and VWD pain management, and on the differences in pain perception between patients, caregivers and providers. As chronic pain often begins at an early age, optimal pain management should include acknowledging patient complaints, exploring pharmacologic and non-pharmacologic options, and optimizing prophylaxis.

\section{KEYWORDS}

acute pain, chronic pain, haemophilia, non-pharmacologic treatment, opioids, von Willebrand disease 


\section{INTRODUCTION}

Recurrent bleeding in patients with bleeding disorders may contribute to progressive joint damage and debilitating joint conditions and results in pain by early adulthood, emphasizing the importance of initiating prophylactic factor replacement therapy at an early age in patients with severe disorders. ${ }^{1,2}$ Differentiating between acute and chronic pain is crucial for determining proper therapeutic strategies. Multiple sources in the literature as well as anecdotal evidence from haemophilia professionals indicate that patients struggle to distinguish between acute pain and persistent daily pain. ${ }^{3-5}$ Since nearly $40 \%$ of patients with moderate or severe haemophilia in one study also indicated their pain was not well treated, ${ }^{3}$ ongoing research is needed to identify specific areas of focus to improve pain management for patients.

Pain perception is complex and highly individualized, often dependent on diverse physical, emotional, cultural and social factors. The absence of validated pain assessment scales for haemophilia has further contributed to the lack of understanding in this population. ${ }^{6}$ Equally important are caregiver and provider perceptions of pain. Caregivers (ie parents) often are the primary source of pain assessment in children, although evidence suggests their assessments are not always accurate. ${ }^{7}$ The gold standard and primary source for identification of pain is the patient. ${ }^{8,9}$ Provider assessments often underestimate patients' pain, especially in those with moderate to severe levels of pain. ${ }^{10-12}$ Differences in perception and communication that exist between patients, caregivers and providers surrounding pain and pain management may influence therapeutic decision-making and, subsequently, patient outcomes.

Despite the clinical significance of pain in bleeding disorders, scarce data exist to describe pain perception among patients, caregivers and providers. The goal of this study was to investigate agreement and differences in the perception of pain and pain management between adolescents and young adults (AYAs) with haemophilia or von Willebrand disease (VWD) and their caregivers and haemophilia treatment centre (HTC) providers.

\section{2 | MATERIALS AND METHODS}

\section{1 | Study population and recruitment}

Data for this study were obtained from the larger study, the Interrelationship Between Management of Pain, Adherence to Clotting Factor Treatment, and Quality of Life (IMPACT QoL). ${ }^{13}$ For this study, we evaluated the perception of agreement between patients, caregivers and providers regarding pain, treatment of pain and perception of pain control. Eligible participants were aged 1325 years; able to read, write and speak English; had haemophilia A or $\mathrm{B}$, or VWD; and provided written consent (parental consent if $<18$ years). Recruitment occurred at major haemophilia conferences, including the annual National Hemophilia Foundation meeting, state consumer meetings, and inhibitor summits and through a Facebook page dedicated to this study from April 2012 to December 2012
All surveys were completed via Survey Monkey (Palo Alto, CA) onsite using Apple iPads (Cupertino, CA). The Munson Medical Center Internal Review Board (Traverse City, MI) approved the study. Significant others (ie a parent, wife, husband or live-in partner) were generally present with the participant and completed a similar survey. The participant or adult caregiver identified their HTC provider and signed a release form. This signed release allowed the surveyors to contact the HTC provider and allowed that provider to complete an online survey matched to the patient. All data were matched from participant to caregiver and HTC provider and were de-identified (other than to the statistician) to maintain anonymity. E-mail reminders were sent to HTC staff members who did not respond to the initial request.

\section{2 | Measurements}

\subsubsection{Acute and chronic pain}

Pain was measured via the Faces Pain Scale-Revised (FPS-R), which comprises six faces illustrating pain intensity: no pain (0), mild pain (2-4), moderate pain (5-6), severe pain (7-8), very severe pain (9) and worst pain possible (10). ${ }^{14}$ Reliability and validity of the FPS-R are established from as young as 6 years up to adults. Pain questions were divided into two separate sections, one for acute and one for chronic/persistent. Acute was described as, "When you are having a bleed, what is your average or usual pain level? This is the type of pain you would have if you were to hurt yourself and bleed into a joint." Chronic/persistent was described as "pain that you have every day or almost every day, and that always or almost always seems to be there even when you are not having a bleed at that moment." Specific questions aimed to identify the following: level of acute and chronic pain; perception of pain control; effectiveness of pain management strategies; need for more pain medication than prescribed or more often than prescribed; and how well providers listened to the participants regarding pain issues.

\subsection{2 | Medications and non-pharmacologic interventions}

Additional questions were asked regarding pharmacologic and nonpharmacologic methods used to manage pain and how well providers and caregivers helped to manage their pain. For each medication, patients and caregivers were asked separately if the patient ever used the medication for acute and/or chronic pain management; providers were not asked to make a distinction between acute and chronic pain with regard to prescribed pain medications. Medications were then divided into seven subcategories to aid in interpretation.

Patients, caregivers and providers were asked about the frequency of the patient using or (in the case of the provider) recommending 24 different non-pharmacologic interventions (eg exercise, ice) for pain. Patients and caregivers were also asked about the patient's use of alcohol, marijuana and illegal drugs other than marijuana. While the patient survey distinguished between acute and chronic pain for each of the non-pharmacologic interventions, caregiver and provider 
survey questions did not make this distinction for non-pharmacologic interventions. Therefore, both possible scenarios were evaluated and are referred to as "assuming acute" and "assuming chronic." Rest, ice, compression, and elevation (RICE) were also compared with nonRICE interventions. Other self-reported data included information about participants' age, sex, self-reported race/ethnicity, health insurance status/type and educational levels of the participants' parents. Additionally, data were collected regarding bleeding disorder type (haemophilia A or B, or VWD), presence of inhibitor and bleeding disorder severity (mild, moderate or severe).

\section{3 | Statistical analysis}

Agreement between patient and caregiver, patient and provider, and caregiver and provider responses was evaluated using the kappa statistic and percent agreement (Stata 14.1; StataCorp LP, College Station, TX). Agreement was rated as poor (kappa<0.2), fair (0.2-0.39), moderate (0.4-0.59), good (0.6-0.79) or very good (0.8-1.0). When the distribution of scores was not similar between groups and the majority of ratings were rank-order data, Spearman's Rho was used to evaluate between-group correlations.

\section{3 | RESULTS}

Eighty-nine patient surveys were completed, with a corresponding survey by either a caregiver (39\%), provider (14\%) or both (47\%). Eight Health Resources and Service Administration regions plus Puerto Rico were represented, with the largest representation from the Great Lakes region. Providers included physicians (26\%), nurse practitioners and physician assistants (30\%) and nurses (44\%). Patients were primarily White males, and the majority had haemophilia A (Table 1). Forty-five surveys evaluated the triad of patient, caregiver and provider.

Overall, kappa on all measures ranged from poor to moderate in each area evaluated: perception of pain and use of pharmacologic and non-pharmacologic interventions (Figures 1-3). Percent agreement on individual measures ranged from $21 \%$ to $100 \%$.

\section{1 | Pain agreement}

Acute pain was reported as at least $4 / 10$ by $55 \%$ of patients, while chronic pain was reported as at least $4 / 10$ by $40 \%$ of patients. Agreement between patients and caregivers (providers were not queried about acute pain) on pain ratings during acute bleeding pain episodes was fair (Figure 1; kappa $=0.27 ; 42.9 \%$ agreement), with patients more likely to report milder pain intensity than caregivers and the highest percentage of patients (29.2\%) reporting moderate pain during a bleeding event (Figure 4A). Acute pain levels were positively correlated between patients and caregivers (Rho=0.49, $P<.01$ ).

There was poor agreement on chronic pain between patient and caregiver (kappa=0.04; 29\% agreement) and patient and provider
TAB LE 1 Patient demographics and clinical characteristics

\begin{tabular}{|c|c|}
\hline Parameter, $\mathrm{n}(\%)$ & Patients $(\mathrm{N}=89)$ \\
\hline \multicolumn{2}{|l|}{ Age, years } \\
\hline $13-17$ & $47(53)$ \\
\hline $18-25$ & $42(47)$ \\
\hline \multicolumn{2}{|l|}{ Sex } \\
\hline Male & $75(84)$ \\
\hline Female & $14(16)$ \\
\hline \multicolumn{2}{|l|}{ Race } \\
\hline White & $63(71)$ \\
\hline Non-White ${ }^{a}$ & $26(29)$ \\
\hline \multicolumn{2}{|l|}{ Type of health care provider } \\
\hline Physician & $14(26)$ \\
\hline NP/PA & $16(30)$ \\
\hline RN/BSN/MSN & $24(44)$ \\
\hline \multicolumn{2}{|l|}{ Primary bleeding disorder } \\
\hline Haemophilia A & $66(74)$ \\
\hline Haemophilia B & $8(9)$ \\
\hline Von Willebrand disease & $15(17)$ \\
\hline Type 1 & $9(10)$ \\
\hline Type 2 & $2(2)$ \\
\hline Type 3 & $4(4)$ \\
\hline \multicolumn{2}{|l|}{ Haemophilia severity } \\
\hline Mild & $9(53)$ \\
\hline Moderate & $1(6)$ \\
\hline Severe & $7(41)$ \\
\hline \multicolumn{2}{|c|}{ Inhibitor to factor/history of inhibitor } \\
\hline Yes & $32(36)$ \\
\hline No & $53(60)$ \\
\hline Did not know & $4(4)$ \\
\hline
\end{tabular}

${ }^{a}$ Most (16\%) non-White respondents were Black/African American, 3\% were of mixed race, and $1 \%$ were Asian. Not all respondents answered all questions.

(kappa $=-0.07 ; 21.4 \%$ agreement) (Figure 1). While no patients reported a total absence of chronic pain $(0 / 10)$, nearly $30 \%$ of caregivers and more than $47 \%$ of providers believed patients had a total absence of chronic pain (0/10) (Figure 4B). Providers also documented the patients' reported average chronic pain level at previous office visits as $0 / 1$, or no chronic pain (51\%; as reported by the patient to the provider during their office visit). Conversely, while $20 \%$ of patients reported very severe/worst possible chronic pain (8/10-10/10), only $6 \%$ of caregivers and no providers perceived the patient experienced chronic pain at that level, and none $(0 \%)$ was reported during previous office visits.

The patient's average chronic pain level was positively correlated with acute pain level during a bleeding event (Rho=0.49; $P<0.01$ ), indicating that high chronic pain was reported with high acute pain, and conversely, low chronic pain was reported with low acute pain. Both the provider assessment of chronic pain and provider documentation 
How well provider listens about pain issues

Need for pain medication more often than prescribed (PT/CG)/provider believes patient takes pain medication more often than prescribed

Need for pain medication more often than prescribed

Patient need for more medication than prescribed (PT/CG)/provider believes patient takes more medication than prescribed (PROV)

Effectiveness of pain management

Need for more medication than prescribed

Perception of pain control

Chronic pain level

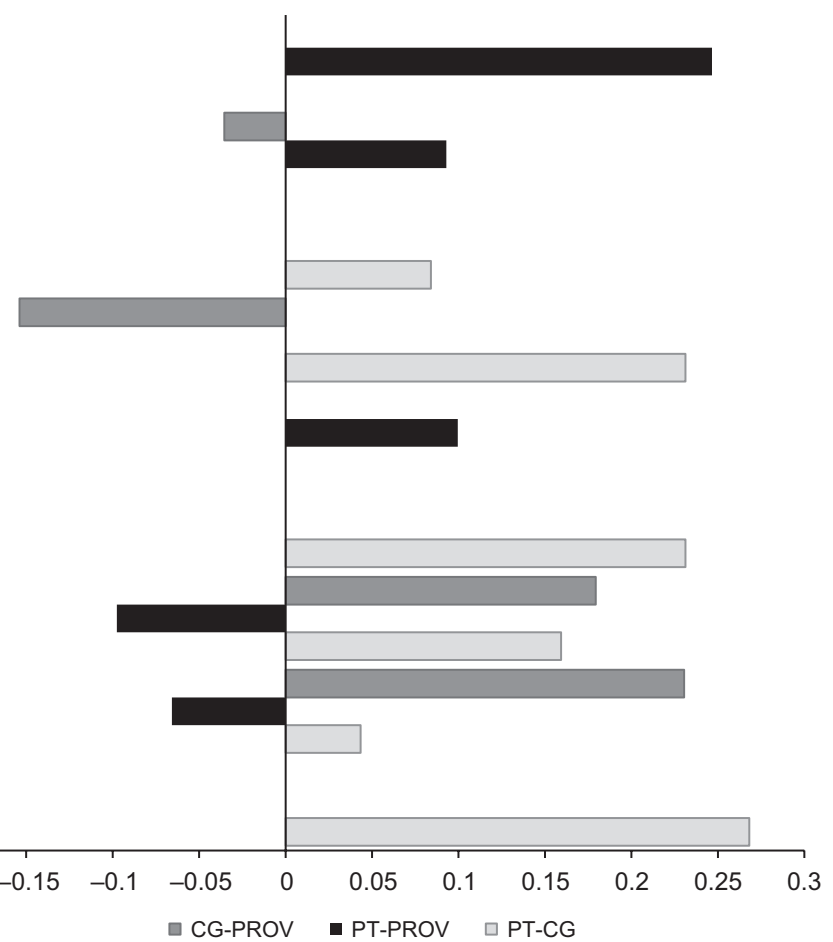

FIG URE 1 Agreement (Kappa) on perceptions of pain and pain management. Agreement was rated as poor (<0.2), fair (0.2-0.39), moderate (0.4-0.59), good (0.6-0.79) or very good (0.8-1.0). CG, caregiver; PROV, provider; PT, patient

of patient-reported chronic pain were positively correlated with patient reports of acute pain (Rho=0.33 for both; $P=0.057)$ but not with patient reports of chronic pain levels.

Patients, caregivers and providers were asked if the patient was in control of their pain or whether pain was controlling the patient (Table 2). Approximately two-thirds of patients, caregivers and providers felt the patient was in control of their pain. In $36 \%$ of patients, $31 \%$ of caregivers and $35 \%$ of providers, pain was reported to be in control of the patient or they were unsure.

When asked to rate the providers' listening skills regarding pain issues, only fair agreement was noted between patients and providers (Figure 1; kappa $=0.25,42.59 \%$ agreement; Table 2). Patients reported a wider range of provider listening than did providers. In $18 \%$ of patients, provider listening was rated as poor or fair compared with $11 \%$ of providers who rated their listening as fair. No provider rated their listening as poor. Only $24 \%$ of providers rated their listening as excellent compared with $39 \%$ of patients who felt their provider had excellent listening.

\section{2 | Medication agreement}

When the provider was asked, "What treatments, other than pain medicine, have you prescribed or recommended for pain management for your patient?" All respondents recommended factor. A large majority of caregivers $(90 \%)$ reported that patients utilized factor, but they were not asked to differentiate between use for chronic vs acute pain; patients self-reported utilizing factor for both acute pain (93\%) and, to a slightly lesser extent, chronic pain (81\%).
The average number of subcategory medications used for acute pain was 1.98 and chronic pain was 2.57. However, one patient acknowledged having tried 12 of the 19 medications options for chronic pain and 17 for acute pain. Although approximately one-third of patients, caregivers and providers did not feel the patient was in control of their pain, fewer believed patients either needed (or took) more medication than prescribed. Fewer caregivers than patients (15\% vs $26 \%$ ) felt the patient needed more medication than prescribed compared with providers, who believed $14 \%$ of patients took more medication than prescribed. In comparison, $26 \%$ of both patients and caregivers felt medication was needed more often than prescribed, while providers felt $11 \%$ of patients took pain medication more often than prescribed. The agreement between individual patients and providers on this measure was poor (kappa $=0.00$ for more medication and 0.09 for medication more often), and was only fair between patient and caregiver (kappa=0.23 for more medication, 0.08 for medication more often).

While $100 \%$ of patients reported acute pain, only $67 \%$ reported utilizing acute pain medication. Only 43\% (20/47 patients) who indicated they have chronic pain reported utilizing chronic pain medications. The average age at which patients began taking medications for chronic pain was 11.5 years.

The most common medications patients reported using for acute pain were factor and acetaminophen, and for chronic pain were factor and non-steroidal anti-inflammatory drugs (Figure 2). With regard to short-acting opioids, patients reported a higher rate of use for chronic pain compared with providers ( $21 \%$ vs $13 \%$ ). The same was seen for long-acting opioids in chronic pain use (patients $11 \%$ vs providers $6 \%$ ). 


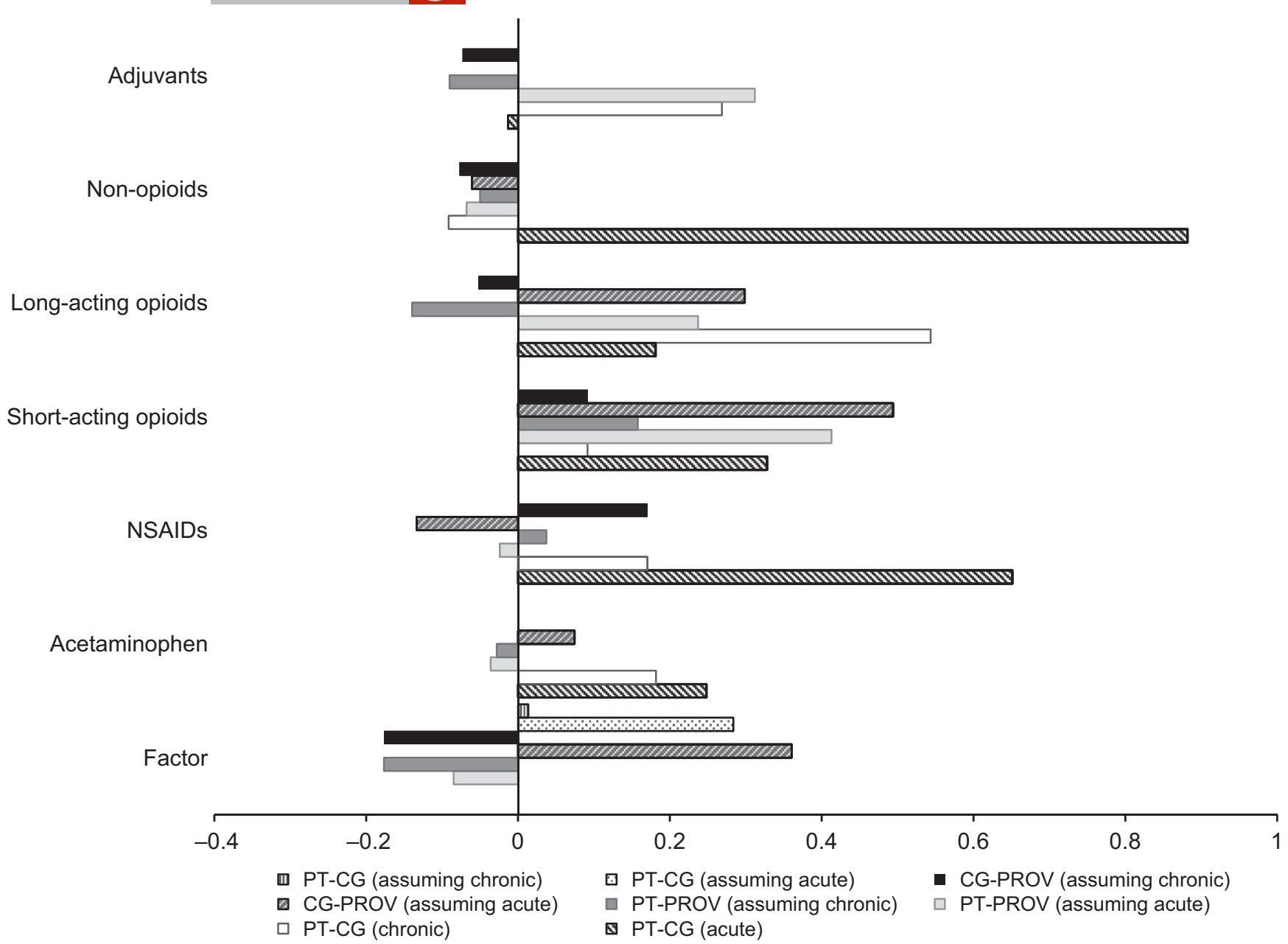

FIGURE 2 Agreement (kappa) on medications used for acute and chronic pain. Patients and caregivers reported use of medications for both acute and chronic pain management. Providers did not provide a distinction between acute and chronic pain; therefore, agreements are assessed by assuming acute and assuming chronic. No distinction was made between acute and chronic pain on the caregiver survey for factor use; therefore, caregiver agreements are assessed by assuming acute and assuming chronic. Agreement was rated as poor $(<0.2)$, fair $(0.2-0.39)$, moderate (0.4-0.59), good (0.6-0.79) or very good (0.8-1.0). CG, caregiver; PROV, provider; PT, patient

The highest kappa values for agreement between patients and providers were on the use of short-acting opioids, assuming either acute or chronic pain, and factor, assuming acute pain.

\section{3 | Non-pharmacologic treatment agreement}

The 10 most frequent non-pharmacologic interventions used by patients are in Figure 3. Use of RICE vs non-RICE interventions was compared between patient report, caregiver observation and provider prescription. Although patients used RICE often, they were also much more likely to use additional non-RICE methods for either acute (chi square=92.36; $P<.0001$ ) or chronic (chi square=65.76; $P<.0001$ ) pain than specifically prescribed by providers. The most frequently used non-RICE methods reported by patients were diversional activities, such as: watching movies or television, thinking about something else, video games, telling jokes, deep breathing and exercise. Although not recommended by providers, alcohol (acute, 9\%; chronic, 14\%), marijuana (acute, 11\%, chronic, 14\%) and illegal drugs (acute, 1\%; chronic, $4 \%)$ were used by patients for both chronic and acute pain.

\section{4 | DISCUSSION}

The factors that influence pain perceptions in the bleeding disorders community among patients, caregivers and practitioners are complex and multifactorial. However, a greater understanding of how these groups describe pain and select therapies for acute and chronic pain is critical to improving the clinical management of bleeding disorders. To date, data on pain perceptions in these groups are limited. The results of this survey demonstrate that there is dissonance among patients, caregivers and providers regarding the perception of pain and the effectiveness of pain management.

Specifically, our findings show there is poor agreement between patients and caregivers across all levels of pain, perception of pain control and effectiveness of pain management. Of note, there was poor agreement between patient and both caregiver and provider on the level of chronic pain, whereas fair agreement was observed between caregivers and providers. It is possible that patients may self-report pain more frequently in an anonymous survey than during an office visit with their health care provider, leading to some of the noted 


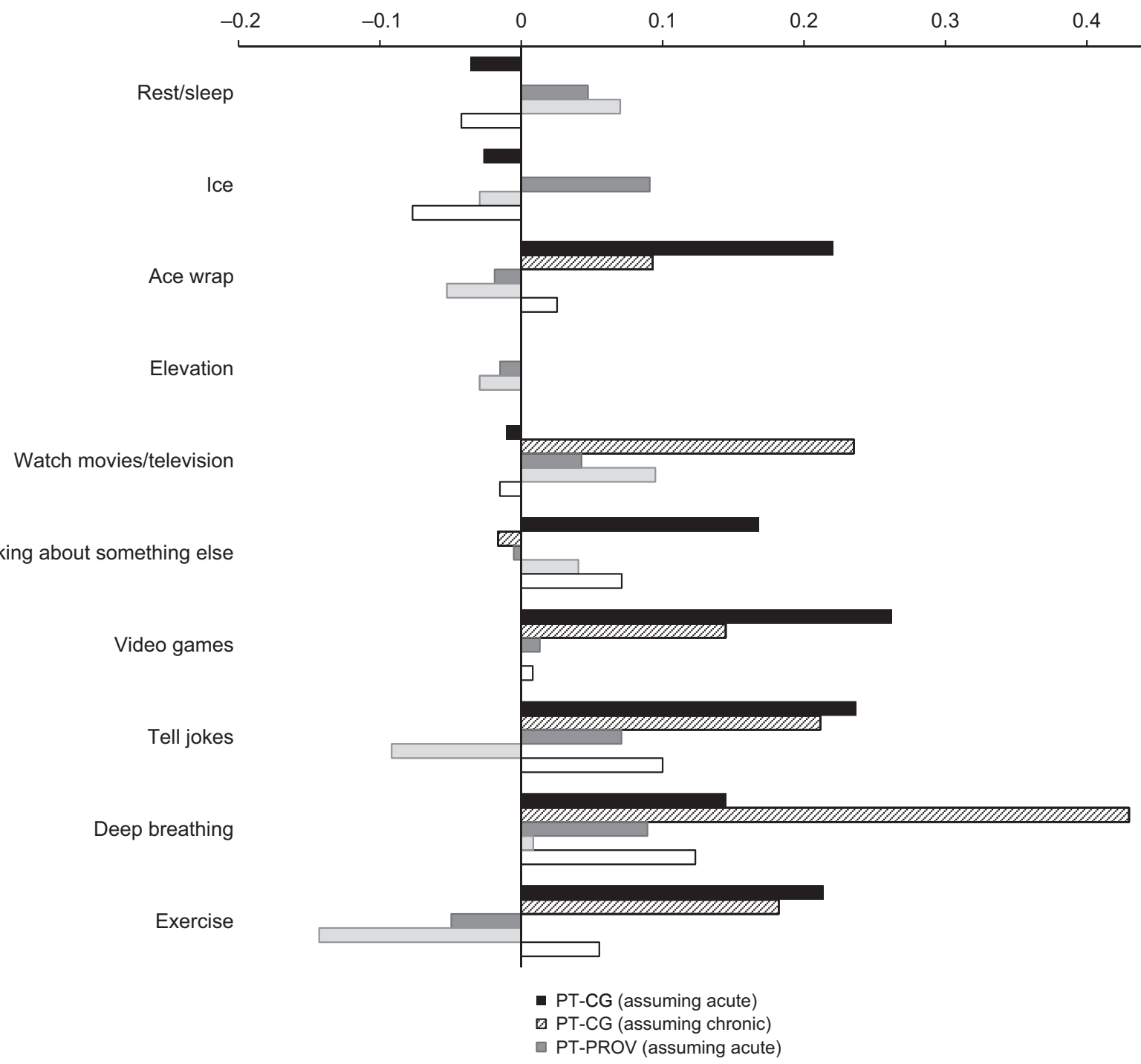

FIGURE 3 Agreement (kappa) on top 10 patient-reported non-pharmacologic interventions used for management of acute and chronic pain. Patients reported using interventions for both acute and chronic pain management. Caregivers and providers did not provide a distinction between acute and chronic pain; therefore, agreements are assessed by assuming acute and assuming chronic. Agreement was rated as poor (<0.2), fair (0.2-0.39), moderate (0.4-0.59), good (0.6-0.79) or very good (0.8-1.0). CG, caregiver; PROV, provider; PT, patient

misperceptions. Anecdotally, patients have told providers that, while they do experience chronic pain, they will not let anyone know the degree of their discomfort as this may result in further limitations to activity, and this may be a reflection of that observation. Patients should be encouraged to speak candidly with their caregivers and providers regarding their pain and the effectiveness of their pain management.

Although not specific to bleeding disorders, the literature demonstrates the influence of caregiver and provider perceptions on patient pain. From the caregivers' perspective, parents who experienced more of their own pain conditions and who were treated more frequently for pain encouraged pain expression in their adolescents and were more likely to catastrophize their adolescent's pain symptoms. ${ }^{15}$ From the providers' perspective, "pain miscalibration," or the difference between the physician's pain rating and the patient's pain rating, has been reported. ${ }^{16}$ Chronicity also affects pain miscalibration among providers, with chronic pain viewed as being less severe than acute pain. ${ }^{12}$
Additionally, the manner in which patients display their pain influences provider assessment and pain medication dosing. ${ }^{17}$ Thus, the level of pain reported may be influenced by many factors and often varies between patients, caregivers and providers. The average level of acute and chronic pain reported by the participants in the current survey was similar to those previously reported. ${ }^{5,17}$ These observed differences in pain perception between patients, providers and caregivers underscore the need for continual education on pain perceptions and the recognition of poor communication between these parties.

Despite $33 \%$ of acute pain patients and $57 \%$ of chronic pain patients not using medications for their specific pain, this survey analysis revealed patients are still using more opioid medication than expected by providers. Although HTC prescribers provide the majority of care, nearly one-third of patients are receiving their pain medications from their primary care provider or from other sources. ${ }^{3,4}$ Given the discrepancy between patient and provider reports of chronic pain, it is 

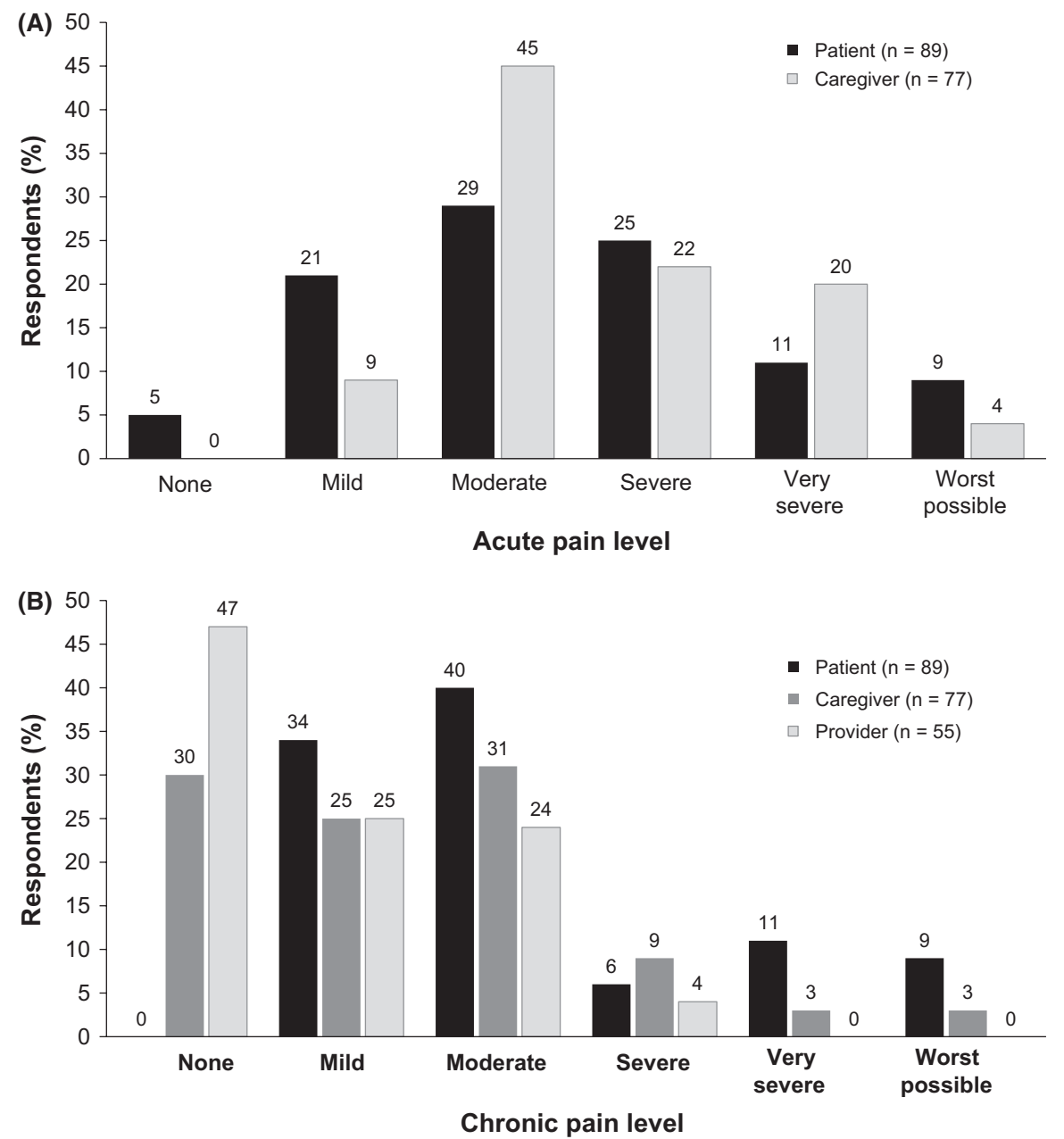

\begin{tabular}{|c|c|c|c|}
\hline Variable, n (\%) & $\begin{array}{l}\text { Respondent } \\
\text { reported }(n=89)\end{array}$ & $\begin{array}{l}\text { Caregiver assessment } \\
(\mathrm{n}=77)\end{array}$ & $\begin{array}{l}\text { Provider } \\
\text { assessment }(n=54)\end{array}$ \\
\hline \multicolumn{4}{|c|}{ Perception of pain control } \\
\hline $\begin{array}{l}\text { Patient in control of } \\
\text { pain }\end{array}$ & $57(64)$ & $53(69)$ & $35(65)$ \\
\hline Not sure & $20(22)$ & $6(8)$ & $14(26)$ \\
\hline $\begin{array}{l}\text { Pain in control of } \\
\text { patient }\end{array}$ & $12(14)$ & $18(23)$ & $5(9)$ \\
\hline \multicolumn{4}{|c|}{ How well provider listens about pain issues } \\
\hline Poor & $7(8)$ & - & $0(0)$ \\
\hline Fair & $9(10)$ & - & $6(11)$ \\
\hline Well & $14(16)$ & - & $16(30)$ \\
\hline Very well & $24(27)$ & - & $19(35)$ \\
\hline Excellent & $35(39)$ & - & $13(24)$ \\
\hline
\end{tabular}

FIGURE 4 Perception of acute and chronic pain during bleeding events. Patients and caregivers reported their perception of acute pain (A); providers were not queried about acute pain. Patients, caregivers and providers reported their perceptions of chronic pain (B)

TABLE 2 Perception of pain control and provider listening possible that some patients may be obtaining opioid medication from other providers, without informing their HTC provider. There are also patients with excellent adherence and good rapport with their providers who may believe they need more medication, but who actually do not seek to take more medication. This survey did not elicit the reasons driving these different behaviours in patients.
Among the participants surveyed, the average age at which patients began taking medication for chronic pain was 11.5 years, suggesting prophylaxis to control bleeding events may be suboptimal or treatment adherence is lacking. ${ }^{13}$ As expected, providers strongly encouraged factor replacement as a treatment strategy for acute pain associated with a bleed. For acute pain management, the AYA 
participants of this study followed that advice better than older adult participants in previous studies; however, the same cannot be said with regard to chronic pain. It was surprising that this age group continued to report pain despite the use of ongoing prophylaxis of factor replacement from an early age, underscoring the importance of adherence and optimal dosing to minimize breakthrough bleeding which leads to acute and thus chronic pain. ${ }^{2-4}$ Compared with $58 \%$ of adults who use factor for chronic pain in the National Pain Study, ${ }^{3} 81 \%$ of AYAs in this study use factor inappropriately for what they identify as chronic pain. Among AYAs with moderate or severe haemophilia, better adherence to prophylaxis is associated with a lower likelihood of having high levels of chronic pain. ${ }^{13}$ The current findings suggest a need for improved adherence to prophylaxis with prescribed clotting factor treatment regimens (either prophylactic or on demand), with the goal to eliminate bleeding events that result in chronic pain.

\section{1 | Study limitations}

When interpreting the results of this study, a few limitations should be considered. First, due to the small sample size of the analysed cohort, survey answers that were on a frequency scale (ie never, rarely, sometimes, often, always) were dichotomized for the comparative analysis on use of the medications and non-pharmacologic treatments. Direct comparisons of survey answers between patients, providers and caregivers were complicated because of differences in survey questions. For example, patients and caregivers were asked if they believed the patient needed pain medication more often and/or more frequently than prescribed, whereas the corresponding provider questions asked whether the provider believed the patient was taking more medication than prescribed, or if the patient was taking medication more often than prescribed. In addition, patients were not asked who prescribed their pain medications. While the data suggest that patients were receiving more pain medication than their treating provider was aware of, there is no clear indication of the source of that medication from the survey. Further, providers were not asked to distinguish between acute and chronic pain for medication and non-pharmacologic treatments, and caregivers were not asked to distinguish between acute and chronic pain for non-pharmacologic treatments, limiting direct comparisons. This study may also be limited by sampling bias, as patients active in the haemophilia community were more likely to participate. Lastly, several of the baseline demographic data (ie bleeding order severity, history of inhibitors) were self-reported by the patients and were not confirmed with the providers.

\section{5 | CONCLUSIONS}

This study provides insight into the clinical management of pain in AYAs with haemophilia or VWD and how perceptions of pain management differ between patients, caregivers and providers. This is important because the average age of chronic pain was 11.5 years, suggesting that pain is an early and common aspect of the haemophilia disease ensemble. There appears to be a disconnect regarding chronic pain perception in that all patients reported chronic pain, while nearly $30 \%$ of caregivers and more than $47 \%$ of providers thought chronic pain was absent. Based on the observation that medication use reported by patients and providers differed, emphasis should be placed on listening to patients regarding their complaints of pain, exploring optimal pain management options to manage pain, and optimizing prophylaxis to minimize or eliminate bleeding events.

In previous surveys, haemophilia patients struggled to differentiate between acute and persistent pain, selecting similar words to describe their pain experience, regardless of the type of pain, which may lead to a failure to select the most appropriate therapeutic option., 4 Additionally, all patients in this analysis reported using factor to treat pain (93\% acute, $81 \%$ chronic). Recent advances in point-of-care ultrasound for haemophilia may hold promise to assist providers and patients in distinguishing between chronic arthropathic pain (treatment with analgesics not factor) and chronic pain due to a prolonged bleeding event (extended treatment with factor). ${ }^{18}$

At every visit, providers should also perform medication reconciliation to understand patient medication history and currently prescribed pain medications. Providers should continue to make greater efforts to discuss patient pain levels, rather than assume an absence of pain if not articulated by the patient, who may accept pain as routine.

Further studies on differences in the perception of pain management in bleeding disorders between patients, caregivers and providers that include a wider age range of patients and a larger sample size are warranted to support the findings of this initial analysis.

\section{ACKNOWLEDGEMENTS}

The authors thank John M. McLaughlin, PhD, of Pfizer Inc for his participation in the development of the original survey and for previous statistical analysis. The authors also gratefully acknowledge the study participants who took the time to complete the surveys. Kathy Covino, PhD, and Bina Patel, PharmD, of Peloton Advantage, Parsippany, NJ, provided medical writing and editorial support, which were funded by Pfizer Inc.

\section{DISCLOSURES}

This study was sponsored by Pfizer Inc. A. Lambing, C.D. Nichols and J. Munn have no potential conflicts to disclose. T.L. Anderson and B.J. Tortella are employees of Pfizer Inc and may own stock/options in that company. M.L. Witkop has received a research grant from Pfizer Inc., at the time of this study, A. Lambing employed at Henry Ford Health System. Detroit Michigan; At the time of this study, M. Witklp employed at Munson Medical Center, Traverse City.

\section{AUTHOR CONTRIBUTIONS}

MLW, JM, BJT and AL contributed to the study design and were study investigators. MLW, AL and JM enrolled patients, participated in the collection and assembly of data and provided data analysis. All authors had full access to the de-identified data, and all authors contributed to 
the drafting, critical review, and revision of the manuscript, with the support of medical writers provided by Pfizer Inc. All authors granted approval of the final manuscript for submission.

\section{REFERENCES}

1. Pipe SW, Valentino LA. Optimizing outcomes for patients with severe haemophilia A. Haemophilia. 2007;13(suppl 4):1-16.

2. Srivastava A, Brewer AK, Mauser-Bunschoten EP, et al. Guidelines for the management of hemophilia. Haemophilia. 2013;19:e1-e47.

3. Witkop M, Lambing A, Divine G, Kachalsky E, Rushlow D, Dinnen J. A national study of pain in the bleeding disorders community: A description of haemophilia pain. Haemophilia. 2012;18:e115-e119.

4. Witkop M, Lambing A, Kachalsky E, Divine G, Rushlow D, Dinnen J. Assessment of acute and persistent pain management in patients with haemophilia. Haemophilia. 2011;17:612-619.

5. Forsyth AL, Witkop M, Lambing A, et al. Associations of quality of life, pain, and self-reported arthritis with age, employment, bleed rate, and utilization of hemophilia treatment center and health care provider services: Results in adults with hemophilia in the HERO study. Pat Prefer Adher. 2015;9:1549-1560.

6. Auerswald G, Dolan G, Duffy A, et al. Pain and pain management in haemophilia. Blood Coagul Fibrinolysis. 2016;27:845-854.

7. Chambers CT, Reid GJ, Craig KD, McGrath PJ, Finley GA. Agreement between child and parent reports of pain. Clin J Pain. 1998;14:336-342.

8. Herr K. Pain in the older adult: An imperative across all health care settings. Pain Manag Nurs. 2010;11:S1-S10.

9. Pasero C, McCaffery M. Pain Assessment and Pharmacologic Managment. St. Louis, MO: Mosby Inc; 2011.

10. Prigent E, Amorim MA, Leconte P, Pradon D. Perceptual weighting of pain behaviours of others, not information integration, varies with expertise. Eur J Pain. 2014;18:110-119.
11. Shugarman LR, Goebel JR, Lanto A, et al. Nursing staff, patient, and environmental factors associated with accurate pain assessment. J Pain Symptom Manage. 2010;40:723-733.

12. Tait RC, Chibnall JT, Kalauokalani D. Provider judgments of patients in pain: Seeking symptom certainty. Pain Med. 2009;10:11-34.

13. McLaughlin JM, Witkop ML, Lambing A, Anderson TL, Munn J, Tortella B. Better adherence to prescribed treatment regimen is related to less chronic pain among adolescents and young adults with moderate or severe haemophilia. Haemophilia. 2014;20:506-512.

14. Faces Pain Scale-Revised Home. Washington, DC: International Association for the Study of Pain, September 14, 2014. www.iasppain.org/FPSR. Accessed: December 16, 2016.

15. Lynch-Jordan AM, Kashikar-Zuck S, Goldschneider KR. Parent perceptions of adolescent pain expression: The adolescent pain behavior questionnaire. Pain. 2010;151:834-842.

16. Marquie L, Raufaste E, Lauque D, Marine C, Ecoiffier M, Sorum P. Pain rating by patients and physicians: Evidence of systematic pain miscalibration. Pain. 2003;102:289-296.

17. Witkop M, Lambing A. Knowledge and attitudes survey in bleeding disorders providers regarding pain. Haemophilia. 2015;21:e465-e471.

18. Ceponis A, Wong-Sefidan I, Glass CS, von Drygalski A. Rapid musculoskeletal ultrasound for painful episodes in adult haemophilia patients. Haemophilia. 2013;19:790-798.

How to cite this article: Lambing A, Nichols CD, Munn JE, Anderson TL, Tortella BJ, Witkop ML. Patient, caregiver, and provider perceptions of pain and pain management in adolescents and young adults with bleeding disorders. Haemophilia. 2017;23:852-860. https://doi.org/10.1111/hae.13293 